\title{
Quality Assurance for Health Checkups and Healthcare Advice with a Particular Focus on the Metabolic Syndrome Program -With a Focus on the Evaluation Criteria Adopted by Healthcare Providers-
}

\author{
Kenta Tezen ${ }^{1)}$, Shigeru Tanaka ${ }^{2)}$ \\ 1) Business Planning \& Development Department, Sompo Japan Insurance Inc. \\ ${ }^{2)}$ Graduate School of Business Administration, Keio University
}

\begin{abstract}
In order to successfully manage the new Japanese Disease Management program, so called "Health checkups and healthcare advice with a particular focus on the metabolic syndrome program", it is essential to implement PDCA cycle into the system. This requires measurement for evaluation. According to our hearing of the eight advanced health insurance societies and two health support service providers, efficiency and continuity seem two key indices for a successful program. Most of the companies and municipal governments seem to pay little attention to these aspects, because of lack of PDCA cycle. It is indispensable to develop sets of indicators to evaluate efficiency and continuity in order to continuously improve the quality of health support activities.
\end{abstract}

Key words: disease management, PDCA cycle, health insurance societies, Japan

\section{Introduction}

The introduction of Health checkups and healthcare advice with a particular focus on the metabolic syndrome program in 2008 has made it possible for non-traditional medical provider to directly contract with health insurance agencies for providing health support services ${ }^{1}$. Many organizations have tried to develop new businesses of health support services ${ }^{2,3)}$.

However, it is worried about that low-priced and low quality services may enter the market. In order to assure quality of new system, it is essential to create a system that will enable the quality of such services to be maintained.

\section{Received: March 31, 2010}

Accepted: April 7, 2010

Correspondence: K. Tezen, Senior Deputy Manager, Business Planning \& Development Department, Sompo Japan Insurance Inc., 1-26-1, Nishishinjuku, Shinjuku-ku, 1608338 Tokyo, Japan

e-mail: KTezen@sompo-japan.co.jp
In this article, the authors analyze the present status of health insurance societies (hereafter referred to as HIS) that have been providing the pioneering activities of quality assurance. Interviews were conducted with two health support service providers and eight HISs. According to the analyses, the authors try to clarify the current problems of the health examination and healthcare guidance system, and then to propose some useful indices for assessment of health support service providers. In order to formulate practical indices, a comparative analysis is also done with the Disease Management Program in the United States of America.

\section{Administration Process of Health Support Services}

The administrative process pertaining to the health examination and health guidance system can be outlined as the following 5 aspects, according to the results of the interviews. 


\section{Planning}

A large proportion of the HISs aims to control the escalating medical expenses due to life style related diseases. In practice, measurable quantities such as the health examination rate, healthcare guidance rate, as well as the rate of decrease and persistency rate of patients, are generally used as targets.

However, whether or not the introduction of the new system will effectively reduce medical expenses due to the metabolic syndrome is not clear for most of the HISs. This is because there are few evidences to show the direct relationship between the decrease in medical expenses and the health examination and healthcare guidance activities at the moment.

Plans are normally made by the HISs themselves, and outsourcing is not considered. External contractors are approached for consultation or outsourcing only for health examinations and healthcare guidance services. Although the effectiveness of these plans is not certain, the planning itself does not yet pose a significant problem.

\section{Health Examination}

In terms of health examinations, different measures are considered for insured persons and for dependents. For insured persons, adding extra items to the current annual examination, which is regulated by the Industrial Safety and Health Law, poses little problem in terms of efficiency. The only problem is the cost sharing resulting from the integration of the annual health examination that employers are obliged to provide, and the specific examination that the insurer is entitled to. Currently, each HIS makes its own adjustments to cost sharing and the division of responsibilities with the cooperation of employer.

On the other hand, dependents will still face to some problems. How to prepare a health examination for the dependents are still a process of trial-and-error.

At present, the HISs, which aim to achieve a certain health examination rate as a mid-term target, have prioritized their responses to deal with insured persons. As the next step in the process, and based on the successful example of other insured persons, the HISs will then focus on improving the examination rate for dependents.

\section{Hierarchization}

The Ministry of Health, Labor and Welfare has set three-tiered levels for healthcare guidance according to the health check-up results: the provision of information, incentive-driven support, and proactive support.

However, some HISs consider that a more detailed level setting is necessary in order to attain better outcome with limited resources. They consider that it is necessary to appropriately allocate resources to each patient according to the more sophisticated analysis based on the accumulated individual data. The health support service providers considered the technique for evaluating health risk of individual client as an important means of differentiating themselves from their counterparts.

HISs considered that health support service providers should equip above mentioned programs as their associate services. Although HISs look for service providers that can consistently perform a range of services, from program planning to project assessment, there are very few providers that can provide the quality services. In fact, it is not easy for health support service providers to organize a quality health education services that fit each person's behavioral characteristics according to results of health examination, under the current situation where the health examinations must be done for a large number of clients within a short period for economic reason.

\section{Health guidance (specific health guidance)}

As like as health examinations, HISs plan to provide health guidance services for insured persons and dependents separately. In the case of insured of HISs, health guidance services are required by the Industrial Safety and Health Law. However, as the health guidance is not obligatory, this service is not always provided for the insured. Especially the workers of small and medium size of enterprises show a very low percentage of receiving health education, even though they need such supports.

Although the new health support system covers middle and old age population between 40 and $74 \mathrm{yr}$ of age, the main target is the middle age working population under $60 \mathrm{yr}$ old. Usually they have relatively low risk of suffering from metabolic syndrome related diseases, it is relatively difficult to efficiently give them incentive to change their unhealthy lifestyle. The most common approach has been to distribute health information about lifestyle related diseases.

The system of health guidance also faces problems in terms of its effectiveness. There are HISs that 
are allowed to use company facilities and manpower, such as conference rooms and occupational health staffs of the company. However, usually they are not allowed to provide services after office hours, on weekends and holidays - all of which are essential as the situation to make it easy for workers to receive the services. Services for dependents face more problems than those for insured persons.

In order to conduct the new program efficiently and pragmatically, there are cooperative arrangements between HISs and medical institutions that allow health education services to be done on the same day of health examination. However, they feel much difficulty to ensure continuous participation of clients. Furthermore, it is a problem how to materialize the quality control system for health examination and health guidance project.

\section{Evaluation}

HISs are required to assume that their contracted service providers conduct self-evaluations, and adopt measures to verify the accuracy of those evaluations. As program evaluation in the health sector is relatively new thing, the methodology of assessment is not established. Thus, ability to perform these assessments is very limited in Japan.

The quality of evaluations depends on the criteria for outsourcing (items to be evaluated) and the data management methods for summarizing the results. In order to conduct proper evaluations of programs, development of appropriate outsourcing criteria is perhaps a necessary condition for the success of the program.

\section{Comparison of US and Japanese Disease Management Programs}

The American Disease Management process and the Japanese specific health examination and healthcare guidance procedure are basically similar programs. However, as Kobayashi indicated ${ }^{4)}$, there are several differences between the two schemes for the following points: objective, target population, and methods.

\section{Objectives}

The objective of typical US Disease Management program is to prevent complications of particular diseases, i.e. diabetes mellitus, asthma, chronic heart dis- eases. On the other hand, the Japanese DM program (the specific health examination and health guidance program) puts more importance on the primary prevention.

\section{Target population}

The target population of the American DM program, is usually much clear and segmented according to pathology and severity. On the other hand, the target population of the Japanese DM project is rather wide ranged from 40 to $74 \mathrm{yr}$ old. As social value is quite different among the different generations, this situation might make it rather difficult to implement a harmonized program that covers entire clients.

\section{Methodology}

As the US Disease Management program intends to prevent the additional medical expenses due to aggravation of pre-existed diseases, the core methodology is the monitoring and forecasting technology and behavioral science to attain a desirable lifestyle modification. As the main purpose of the Japanese Disease Management program is the primary prevention, it seems that much importance is put on behavioral changes such as physical fitness and dietary habit. However, most of them are developed in the Occidental countries not in the Japanese context. It is expected to develop the Japanese original methodology based on the behavioral psychology that would fit for the Japanese culture. Various entertainment devices such as video games are expected as possible solutions for the Japanese DM programs.

\section{Time schedule}

While the target period for achieving these objectives in the US is comparatively short-term, owing to the fact that the target patients are those who have already contracted the disease, Japanese HISs expect results on a relatively medium-term basis.

The Japanese system initially aimed at reducing the number of patients, both actual and potential, with metabolic syndrome related disease, and consequently to lower the number of patients with lifestylerelated diseases such as diabetes and coronary diseases. The effectiveness of such a system must be examined on a medium-term basis, as the reduction of medical expenses is a secondary effect. The current goal of Japanese medical insurance is to create healthy society, modifying lifestyle habits and behavior by 
intervening at an early stage. It seems that the Japanese approach is more ambitious compared with similar programs of other developed countries.

\section{Indices for Evaluation of Efficiency and Continuity of the Program}

Judging from interviews with HISs, it is seen as important to assure the quality of health guidance services, including the method of hierarchization, in order to maintain the overall quality of the entire project of specific health examinations and provision of healthcare guidance.

In order to successfully change the client's lifestyle healthier, it is very important to develop methods for keeping clients to consistently engage in the programs. Modification of behavior is encouraged in three ways: (1) basic health education at the initial stages of guidance; (2) continuous external support through interviews and phone calls; and (3) self-assistance tools to support daily practices.

Although all three components are necessary in order to attain a successful behavior modification, it seems very important to provide tools for self-assistance. The most common tool is the self-management diary of one's daily activities. Based on the recorded information, health professionals can give practical advices to attain one's behavioral goal.

The components that are indispensable for successful modification of one's behavior are efficiency and continuity. Efficiency could be defined as the total volume of modification in one's behavior divided by the cost of achieving it, while continuity could be defined as the percentage of that modification that is sustained.

Efficiency is essential to the continuation of a project funded by limited resources obtained from insurance premiums. Continuity is also important to ensure the overall efficiency. An ideal case of healthcare guidance would be one where the tools continue to be used, and where activities encourage by using the tool become part of one's lifestyle. If this continuity can be achieved, the amount of investment necessary would decrease. Thus economic evaluation method must be developed and implemented into the health support programs.

Efficiency is measured by two indices: past performance record (the number of health guidance sessions that are completed), and targeting capability (the number of items that could be analyzed). Targeting capability is an index that shows whether the accumulated data can be analyzed.

On the other hand, continuity can be divided into two indices: operability and entertainment quality. A high rate of continuity means that the activity has been integrated into one's lifestyle. For this to happen, however, it is essential for the activity to be enjoyable, without having a sense of additional burden.

Operability is an index that measures activities adding little burden to one's lifestyle. Keeping a record of daily activities makes it possible to look back on one's results on a weekly or monthly basis. Just as a household account book plays an important role in economizing on family finances, keeping a similar record is a significant step towards achieving a modification in one's behavior. It is also essential to communicate with one's instructors, and to gather data from the insurer's point of view. In terms of specific indices for analyzing how feasible such recordkeeping is, the following may be used: (1) time required for recording, and (2) frequency of recording.

In order to increase operability of program, one possible solution is to introduce IT measures for keeping these records. Numerical values such as body weight and body fat can be easily measured and recorded by devices. By programming such devices and management tools, such as websites, the burden of recording will be reduced.

As for entertainment quality, people tend to pursue what they personally enjoy, and try to be at ease in their life, outside of their work. Of course, this does not apply to everyone, but certainly a majority of people eats what they like, and avoid unnecessary walking. To modify one's behavior is to change their preferred lifestyle. Evidently, this is not an easy task, and this is why the entertainment quality of the program should be considered. Entertainment quality here should be understood to apply not simply to activities that are interesting and fun, but rather to those which include stimulating or delightful elements, such as an experience involving personal growth, a sense of competition among friends, or praise from someone.

In terms of specific indices, some quantify the reactions of the subjects: (1) number of patients with access to tools, (2) utilization rate of the tool; while others judge its "game" quality: (3) presence of competitive elements, (4) presence of elements that nur- 
ture a sense of personal growth. Still others check whether the activity in question appeals to one's sensitivity: (5) presence of checks at regular intervals, which make one aware of being monitored; (6) visualization (of conditions such as visceral fat); and (7) design quality.

\section{Conclusion}

In order to successfully manage the new Japanese Disease Management program, so called "Health checkups and healthcare advice with a particular focus on the metabolic syndrome program", it is essential to implement PDCA cycle into the system. This requires measurement for evaluation. According to our hearing of the eight advanced HISs and two health support service providers as well as to the comparative study between the American and Japanese systems, efficiency and continuity seem two key indices for a successful program. As the traditional health support activities have been done as a part of welfare services of company and municipal government based on the annual budget, little attention has been paid to these aspects. It is indispensable to develop sets of indicators to evaluate efficiency and continuity in order to continuously improve the quality of system.

\section{References}

1) Matsuda S: Health promotion policy in Japan. APJDM 1, 11-17 (2007).

2) Takimoto M, Kibushi M, Okoshi Y, et al.: Body weight reduction program for metabolic syndrome-evaluation of effect after one-year-intervention-. APJDM 2, 93-96 (2008).

3) Kibushi M, Takimoto M, Okoshi Y, et al.: Body weight reduction program for metabolic syndrome-Introduction of Internet-based HALSMA diet program-. APJDM 2, 119-122 (2008).

4) Kobayashi A: The development of a quality management mechanism in disease management: the US experiences and its suggestions. APJDM 1, 97-102 (2007). 